$\underline{\text { X-ray crystallography }}$

\section{Advantages of electronic 'film'}

from Stephen C. Harrison

BY comparison with other areas of molecular biology, the determination of macromolecular structure has suffered because of the time-consuming and tedious course of X-ray crystallographic investigations. Recent advances in genetic manipulation have made it possible to produce considerable quantities of all sorts of interesting proteins and protein-nucleic acid complexes, some of which have been crystallized. It is therefore of more than casual interest that as a result of progress in the technology of X-ray detectors we appear to be on the verge of achieving a substantial acceleration in the collection of intensity data from crystals. The new detectors are two-dimensional position-sensitive devices which permit the simultaneous recording of large numbers of reflections with the accuracy usually associated with onereflection-at-a-time diffractometers.

The earliest X-ray intensity measurements from crystals were made by following ionization with a gold-leaf electrometer. The hands of Bragg père and Bragg fils, actuated by the beat of a metronome, served as the 'stepping motors' for moving the crystal through its reflecting position. (Bragg junior wrote an entertaining account ${ }^{1}$ of the times in which the intensity of the 100 reflection from $\mathrm{NaCl}$ could be reported ${ }^{2}$ to be ' 676 , when the crystal was turned through five minutes of arc for each beat of the clock'). As more complicated structures came to be studied, recording with photographic emulsions became standard, since it enabled many reflections to be measured sim!ltaneously. The advent of automated diftractometers in the late 1950s turned a large part of the crystallographic community to electronic data collection, using a proportional counter or similar device, but the inefficiency of recording only one reflection at a time made diffractometry a poor choice for larger or more complicated proteins. A simple calculation presented by Arndt ${ }^{3}$ shows that for unit cells larger than about $150 \AA$, appropriate photographic recording is the significantly more efficient approach. Indeed, stepped oscillation or rotation photography has become standard in most laboratories working on large proteins and at synchrotron installations supporting macromolecular crystallography.

Photographic film is actually an excllent detector, and for $1.5 \AA \mathrm{X}$ rays it is about 70 per cent quantum efficient. Automated two-dimensional densitometers, such as the widely used rotating-drum scanners, make digitization of films and evaluation of intensities relatively straightforward, although in a number of cases the tedium of this step has proved rate-limiting. A more significant disadvantage of photographic measurement is the limitation to its accuracy imposed by the small fraction of recording time during which a given reflection is diffracting. In oscillation photography, a $1^{\circ}$ photograph is 'typical', but the angular range over which a particular order diffracts might be $0.1-0.2^{\circ}$, depending on geometrical factors and crystal quality. Background is recorded on the film during the remaining $0.8-0.9^{\circ}$, decreasing the signal-to-noise ratio. This disadvantage could be eliminated by the expedient of recording a series of $0.1^{\circ}$ photographs - and it is precisely this possibility, impractical with actual film, that is offered by the new devices.

Currently there are three quite different two-dimensional detectors for singlecrystal X-ray diffraction, each based on different design ideas and on significantly different technologies. The device developed by $U$. Arndt at the Medical Research Council Molecular Biology Laboratory in Cambridge, and now marketed by Enraf Nonius, records the diffraction pattern on a fluorescent screen connected by fibre optics to an image intensifier ${ }^{4,5}$. The intensifier output is read by a television system linked to a computer. The $\mathrm{Gd}_{2} \mathrm{O}_{2} \mathrm{~S}$ input screen is $47 \times 63 \mathrm{~mm}$ and the final scan is binned at $512 \times 512$ pixels. The size of the screen and the point-to-point resolution imply that data to $3 \AA$ resolution can be collected at a single detector setting from crystals with a $100 \AA$ cell constant. Crystals with larger unit cells require a longer crystal-detector distance and several detector settings. The device includes a 4-circle goniostat to facilitate resetting.

The detector developed by N. Xuong and colleagues at the University of California at San Diego is a multiwire proportional counter, derived from detectors developed for high-energy physics ${ }^{6,7}$. It consists of a xenon-filled ionization chamber with delay-line readout from planes of parallel wires in $x$ and $y$. It has an active area of $27 \times 30 \mathrm{~cm}$, with a spatial resolution of $0.6 \mathrm{~mm}$ full width at half $\max$ imum (FWHM) in the horizontal direction and $2 \mathrm{~mm}$ in the vertical direction. The modest resolution necessitates a large crystal-detector distance (1 m or more), and full recording of a protein diffraction pattern to 3 Åresolution may require multiple settings or an array of more than one detector. The set-up in Xuong's laboratory has already been used to solve several structures, including dihydrofolate reductase ${ }^{8}$ and the large ('Klenow') fragment of Escherichia coli DNA polymerase I (T. Steitz, personal communication). A similar device has been constructed at the University of Virginia.
A different type of xenon-filled detector has been developed by R. Burns of the Xentronics Corporation, Cambridge, Massachusetts. It is also a multiwire device, but with capacitive readout and with much higher spatial resolution $0.2 \mathrm{~mm}$ FWHM in both $x$ and $y$ ). Its active area is $12 \mathrm{~cm}$ in diameter, and it is fitted with a modified oscillation 'camera'. The high spatial resolution permits a single detector to mimic a conventional X-ray film. The Xentronics detector has been used to collect data from several quite challenging crystals, including tomato bushy stunt virus, with a unit cell constant of $383 \AA$, and in our preliminary experience has shown very favourable intensity statistics ${ }^{9}$.

Each of these devices is likely to have its own niche in the ecology of crystallographic applications. An advantage of the television-based design is its inherently higher data-rate capacity. The xenon-filled devices are at present limited by dead time to a total rate (over the face of the detector) of $30-50 \mathrm{kHz}$. Although not a significant disadvantage with conventional sources, this limitation can restrict possible applications with synchrotron sources. The Xenotronics device has the advantage of being a compact unit that can be moved easily from one X-ray set to another. Its large active area and high spatial resolution also give it an edge in the large-unit-cell range.

The gain in signal-to-noise that detectors offer with respect to film can be used either for increased accuracy or for increased speed. The increased accuracy will be especially helpful for medium-to-large unit cells, where reliable heavy-atom differences have sometimes been difficult to measure and noisy difference data difficult to interpret. The increased speed offered by these new approaches to data collection is likely to have immediate impact in the analysis of variants, mutants, modified proteins, active-site complexes and the like. Since these aspects of an investigation follow the initial structure determination, they are usually not held up by the phase problem (for example, the search for heavy-atom derivatives) but rather by the process of intensity measurement itself. It should soon be much simpler to couple sitespecific mutagenesis and $X$-ray crystallography, in order effectively to test hypotheses based on the examination of a new structure.

1. Bragg, W.L. Acta. crystallogr. A25, 1 (1968)

2. Bragg, W.H. Proc. Camb. phil. Soc. 17, 43 (1914).

Arndt. U.W. Acta. crystallogr. B24, 1355 (1968).

Arndt, U.W. Nucl. Instrum. Meth. 201, 13 (1982).

Arndt. U.W. \& Thomas, D.J. Nucl. Instrum. Meth. 201, 21 (1982).

6. Xuong, N., Freer, S., Hamlin, R., Nielsen, C. \& Vernon, W. Acta. crystallogr. A34, 289 (1978).

Hamlin, R. et al. J. appl. Crystallogr. 14, 85 (1981).

8. Matthews, D.A. et al. J. biol. Chem. 253, 6946 (1978)

9. Durbin, R.M., Burns, R., Harrison, S.C. \& Wiley, D.C Proc. ACA Summer Meet., Snolomass, Colorado (1983).

Stephen C. Harrison is in the Department of Biochemistry and Molecular Biology, Harvard University, 7 Divinity Avenue, Cambridge, Massachusetts 02138. 